\title{
LA TEOLOGÍA COMO \\ PEDAGOGÍA DEL ACOMPAÑAMIENTO \\ UNA REFLEXIÓN DESDE EL TRABAJO EDUCATIVO EN EL CONTEXTO DE PANDEMIA
}

\section{THEOLOGY AS A PEDAGOGY OF ACCOMPANIMENT A REFLECTION FROM EDUCATIONAL WORK IN THE CONTEXT OF A PANDEMIC}

\author{
JUAN PABLO ESPINOSA ARCE* \\ Mg. en Teología Fundamental \\ Pontificia Universidad Católica de Chile
}

Artículo recibido el I4 de enero de 202I; aceptado el o7 de julio de 2021.

"jpespinosa@uc.cl

https:/orcid.org/0000-0002-2474-9185

Cómo citar este artículo:

ESPINOSA, J.P. "La teología como pedagogía del acompañamiento. Una reflexión desde el trabajo educativo en el contexto de pandemia" en Palabra y Razón. Revista de Teología, Filosofía y Ciencias de la Religión. № 19 JULIO 202I, pp 55-76 https://doi.org/I0.29035/pyr.19.55 


\title{
RESUMEN
}

La situación de la pandemia nos ha propuesto el desafío de realizar nuestro quehacer académico-pedagógico desde una modalidad online. La distancia física con nuestros estudiantes se ha transformado en una oportunidad para imaginar una teología que, desde su disciplina y cuerpo epistémico, se ubique como un modelo de lo que hemos denominado una pedagogía del acompañamiento. La importancia del cuidado del otro, del autocuidado, de la entrega de reflexiones en el campo de la espiritualidad, de lo humano, de cómo aprender a sobrellevar este desafiante tiempo y de pensar qué lugar ocupa Dios y su presencia en este contexto, están en la base de lo que se busca ofrecer en este trabajo. El objetivo del presente trabajo es ofrecer una reflexión en torno a qué ha significado la educación teológica online, la cual no solo ha entregado categorías de dicha área, sino que ha buscado ser espacio de acompañamiento, diálogo y contención entre los participantes. Desde aquí, en definitiva, quisiera pensar el cómo de la acción de Dios en este tiempo que se puede dejar leer en las preguntas y experiencias de los participantes de las clases.

Palabras claves: acompañamiento / discernimiento / pedagogía / pandemia / vida

\begin{abstract}
The situation of the pandemic has proposed us the challenge of carrying out our academic-pedagogical work from an online mode. The physical distance with our students has become an opportunity to imagine a theology that, from its discipline and epistemic body, is located as a model of what we have called a pedagogy of accompaniment. The importance of caring for the other, of selfcare, of giving reflections in the field of spirituality, of the human, of how to learn to cope with this challenging time and of thinking about what place God occupies and his presence in this context, are in the basis of what you want to offer in this job. The objective of this paper is to offer a reflection on what online theological education has meant, which has not only provided categories of this area, but has also sought to be a space for accompaniment, dialogue and contention among the participants. From here, in short, I would like to think about the how of God's action in this time that can be read in the questions and experiences of the class participants.
\end{abstract}

Keywords: accompaniment / discernment / pedagogy / pandemic / life 


\section{Introducción y propósito del presente desarrollo}

Lo que busco proponer en este desarrollo es pensar el vínculo entre pedagogía y teología a partir del concepto y experiencia del acompañamiento. El tiempo de la pandemia ha significado para cada uno de nosotros un aprender a resignificar las distancias con nuestros estudiantes, con los demás profesores de nuestras Facultades e Institutos, con nuestras familias y amigos. La distancia y la ausencia física, como experiencias fundamentales de la pandemia, son elementos que requieren una suerte de pensamiento a largo plazo. La pedagogía y la teología, en su vínculo entrañable que, personalmente, he aprendido a reconocer, valorar y proponer, deben imaginar cómo vivir, pensar y celebrar en medio de la pandemia, reconocer cuáles son sus alcances, pensar cuáles son sus oportunidades, crisis y contrariedades. Y, a su vez, ayudar a pensar e imaginar cómo será el tiempo de la post-pandemia $y$, de esa manera, recuperar aquellos elementos que caracterizan sus reflexiones en cuanto expresiones de un anhelo más profundo.

Dicho anhelo, quisiera comprenderlo, junto con Viktor Frankl, desde el concepto de la autotrascendencia que busca la voluntad del sentido. El psiquiatra de Viena define el concepto de autotrascendencia indicando que en el hombre y la mujer existe un nivel más alto que la mera respuesta a estímulos, a la satisfacción de deseos o al cumplimiento de tareas. Eso, para Frankl, representa una voluntad de sentido más allá de lo contingente, una mirada a largo plazo. Es ahí donde surge el concepto de la autotrascendencia. En palabras de Frankl:

"la existencia dependía de la "autotrascendencia" (...) así llegué a comprender que el primordial hecho antropológico humano es estar siempre dirigido o apuntado hacia algo o alguien distinto de uno mismo: hacia un sentido que cumplir u otro ser humano que encontrar, una causa a la cual servir o una persona a la cual amar. Tan sólo en la medida en que alguien vive esa autotrascendencia de la existencia humana, es auténticamente humano o deviene auténticamente él mismo".

Para Francesc Torralba, la autotrascendencia de Frankl está íntimamente vinculada con la experiencia de la espiritualidad, pero entendiendo por ella la adscripción a una determinada confesión religiosa, sino que tiene que ver con el desarrollo de las capacidades

I V. FRANKL. Psicoterapia y humanismo ¿tiene un sentido a la vida? FCE: México, 20I4, p.36. 
sutiles que están presentes dentro de cada vida. Para Torralba, "la autotrascendencia es una capacidad singular en el ser humano, que le lleva a superar barreras y adentrarse en terrenos desconocidos, a superarse indefinidamente a sí mismo, a buscar lo que se esconde más allá de los límites de su conocimiento". Somos capaces de autotrascendencia en cuanto somos capaces de salir de nosotros mismos, de estar en vínculo con realidades diversas a lo que cada uno de nosotros es y a experimentar la audacia de reconocer que nuestros marcos interpretativos se enriquecen en el encuentro con la diversidad.

Por lo tanto, si la autotrascendencia sugiere la capacidad de salir de nosotros mismos para ir al encuentro de una realidad otra (sentido que cumplir, encuentro interpersonal, causa por la cual el ser humano se compromete), pienso que tanto la pedagogía como la teología representan espacios de autotrascendencia y de acompañamiento, cuestión que es posible incluso comprender desde la etimología de ambas palabras. Pedagogía significa el arte de llevar o acompañar (agó) a los niños (paideia) ( $\pi \alpha \iota \delta \alpha \gamma \omega \gamma o ́ \varsigma)$ (paidagōgos). Hace referencia al tutor o al que cuida a los niños. Teología, por su parte, indica una reflexión, un logos, un sentido (en términos de Frankl, por ejemplo) sobre Dios en cuanto otro y, a su vez, sobre la reflexión sistemática sobre la experiencia creyente en ese mismo Dios revelado en Jesucristo (Cf. Jn I,I4; Heb I,I-2). En ambos casos, las reflexiones articulan una propuesta centradamente descentrada, es decir, el sujeto y/o la realidad a la que hacen referencia está más allá del sujeto que la enuncia o la practica. En clave cristiana, quisiera comprender cómo aquel Dios del cual tratamos de balbucear una palabra, un sentido, se puede reconocer en las preguntas, en las vivencias y en las búsquedas de nuestros estudiantes. Por ello, es que pienso que la teología debería constituir una pedagogía del acompañamiento. Para acercarme a estas intuiciones, el recorrido planteado tendrá los tres siguientes momentos:

En una primera parte, pensaré el arte del acompañamiento, que es el concepto central de nuestra propuesta. Entenderé el acompañamiento desde su dimensión de concepto bi/faz, es decir, me acercaré a él desde lo pedagógico/espiritual; educativo/teológico; teórico/pastoral. Pienso, con ello que lo polisémico del concepto nos permite ampliar su profundo significado. En segundo lugar, quisiera recuperar algunas formas en las que he ido viviendo este tiempo de educación teológica online o a la distancia y cómo ella ha trabajado no sólo lo teórico de nuestras disciplinas, sino que ha sido un espacio de compañía

2 F. TORRALBA. Inteligencia espiritual, Plataforma Editorial: Barcelona, 2019, p.45. 
entre los participantes. Finalmente, quisiera proponer un concepto: anástasisgogía, es decir, proponer la idea en torno a qué podría significar una pedagogía de la Anástasis, del poner(nos) de pie, una educación teológica que tenga a la Resurrección de Jesús como modelo y sentido de las prácticas humanas presentes. Quizás, la Resurrección de Jesús puede representar un concepto interesante para pensar el cómo de nuestro acompañamiento en este desafiante tiempo que vivimos.

\section{El arte del acompañamiento: compartir la vida con otros}

\section{I Etimología del acompañamiento y la importancia de lo nutricio en la pedagogía}

Carlos Puerta Gil en su artículo "El acompañamiento educativo como estrategia de cercanía impulsadora del aprendizaje del estudiante", indica - haciéndose eco de Gómez de Silva - que el concepto acompañamiento se deriva del latín companio y significa "quien come pan con otro"; en la actualidad, según el mismo autor, tiene un sentido de ir con u ocurrir con"3. Y, más adelante señala:

"el acompañamiento, por tanto, es una acción educativa intencionada que se fundamenta en la cercanía, en la disposición para afectar y dejarse afectar por el otro, teniendo como horizonte primordial la potencialización de sus capacidades, de tal manera que este siga configurando y moldeando sus sueños y esperanzas"4.

Lo interesante de la definición de Puerta Gil es que ubica al acompañamiento como un proceso complejo en el cual convergen emociones (dejarse afectar por otro), la idea de un proyecto común (potenciar las capacidades de otro), incluso la idea de subsistencia material y vida (compartir el pan con otro). Acompañar, por lo tanto, es permitir que la otra persona, aquella con la cual comparto un camino de crecimiento, viva y viva bien. La lógica del bienestar está en la base del acompañamiento. Incluso, esta experiencia podría traducirse también a partir de lo que autores como Patricio Alarcón Carvacho presentan como una pedagogía de carácter nutritivo y muy enfocada en el sentido de dar vida a los que componen el entramado específico de lo pedagógico. Es interesante la evocación de la nutrición aplicada específicamente al sentido del arte pedagógico. Alarcón Carvacho incluso llega a identificar

3 C. PUERTA GIL, "El acompañamiento educativo como estrategia de cercanía impulsadora del aprendizaje del estudiante" en Revista Virtual 49, Septiembre-Diciembre (2016), pp. 2-6, 3.

4 Carlos Puerta Gil, "El acompañamiento educativo", p.4. 
la importancia de contar con lo que él denomina los "profesores nutricios"5, los cuales poseen las características de fortalecer las identidades de los interactuantes tanto como la propia, fortalecimiento que, a juicio de Alarcón, debe realizarse en todas las dimensiones de la existencia personal.

Lo nutritivo, las prácticas del bienestar y las lógicas del cuidadoacompañamiento suponen el establecimiento de una visión integral e integrada de la vida. A la vez, Alarcón Carvacho también coloca especiales énfasis en lo que él denomina "estudiantes nutricios", los cuales son capaces de fortalecer la identidad de los profesores que trabajan con ellos, de sus compañeros y de otros miembros de la comunidad, ya sea educativa, familiar o de amigos. Para Alarcón los estudiantes nutricios se mueven, preferentemente, a partir de dos actitudes rectoras: el involucrar y el enriquecer. La nutrición, con ello, va estableciendo diversas formas a través de las cuales el profesor-estudiante, tanto en su individualidad como en su compleja relación, van desarrollando las prácticas humanas. $\mathrm{Y}$, a la vez, lo nutricio establece una forma sugerente de comprender el acompañamiento, con la cual esbozamos que no solo se comparte el pan físico, sino que se comparte el pan vital, el pan de la escucha, del cuidado y del enriquecimiento mutuo.

\subsection{El comienzo del acompañamiento: las vidas puestas en común en medio de las crisis}

Este enriquecimiento mutuo constituye, a nuestro juicio, la prioridad absoluta de lo pedagógico. No es solo la entrega de una serie de contenidos académicos, sino que es la invitación a reconocer que es en la trama de los acontecimientos vitales donde se va desarrollando el fondo auténtico de la educación, fondo vivido en la compleja interrelación de los miembros de la comunidad educativa. Paulo Freire coloca acentos en esto cuando expresa que en la actualidad "la educación ya no es entendida como formación, sino pura y exclusivamente como entrenamiento"? Formación y entrenamiento aparecen como conceptos opuestos en la reflexión de Paulo Freire. Si entrenamiento está vinculado a una entrega de contenidos desconectados con la compleja realidad en la que se desarrolla el quehacer educativo, la formación supone la

\footnotetext{
5 P. ALARCÓN, Educación y coexistencia: aproximación a una projimología para la felicidad y la salud. Cuarto Propio: Santiago de Chile, 2015, p.I95.

6 P. ALARCÓN, Educación y coexistencia, 200.

7 P. FREIRE, Pedagogía de los sueños posibles: por qué docentes y alumnos necesitan reinventarse en cada momento de la historia. Siglo XXI Editores: Argentina, 20I5, p.43.
} 
capacidad de leer los contextos históricos, sociales y culturales y desde allí ser capaces de pensar los contenidos propuestos por los distintos currículos propuestos en las instancias educativas. Este doble paso, a saber, la lectura del mundo y, en segundo lugar, la lectura de los contenidos específicos es un elemento sugerente al momento de pensar el acompañamiento, en cuanto supone el desafío siempre presente de mostrar un verdadero interés por las distintas cosmovisiones y vidas que se dan cita en un grupo educativo. Y es sugerente en cuanto los miembros de la comunidad educativa se van haciendo conscientes de la importancia de la lectura de los contextos y del mundo. Es una auténtica experiencia de alfabetización en el acompañamiento. Alfabetizar en la práctica del acompañamiento es ir generando la conciencia de que la forma de ser humanos pasa necesariamente por el encuentro transformador entre los seres humanos. Esto es fundamental para Freire, quien lo expresa, a propósito de la educación con niños, desde las siguientes propuestas:

"Esta relación íntima con el ambiente/mundo es la lectura que
antecede y permite la creación de signos y símbolos de los que se
impregnan las instancias de registros para permitir las relecturas.
Relecturas contextualizadas por signos producidos en la lectura
primera del ambiente/mundo y no por signos y símbolos típicos
de los libros para enseñar a leer. Esto nos brinda una enseñanza
sumamente importante. En general, en nuestra sociedad los niños
son sometidos a un proceso pavoroso de imposición de signos a
priori, sin que se establezca ninguna relación entre esos signos y
lo que los niños vivencian leyendo el mundo y representándolo
para sí en símbolos asociados a esa lectura anterior de la palabra
escrita".

En el planteamiento de Freire se destacan las intuiciones de la lectura del mundo como antecedente a la lectura de los contenidos propuestos. El acompañamiento pedagógico que debe comenzar a formularse pasa por el reconocimiento de los signos que el estudianteprofesor va aprehendiendo del ambiente. Ellos son el terreno sobre el cual se van desarrollando las categorías propias del mundo académico. Este diálogo entre lo que podríamos llamar el mundo conocido y el mundo de los relatos del texto pareciera surge como el modo adecuado de generar una sana práctica del acompañamiento. Vamos acompañándonos desde la puesta en común de nuestros propios mundos personales, desde nuestras modulaciones familiares, desde los conocimientos previos que

8 P. FREIRE, El maestro sin recetas: el desafío de enseñar en un mundo cambiante. Siglo XXI Editores: Argentina, 20I6, p.II9. 
hemos ido adquiriendo en la experiencia de vivir en contacto con otras realidades.

Esto, pienso, viene a humanizar el acompañamiento mismo y a no concebirlo como una práctica meramente programada, sino que surge incluso como una propuesta marcada por lo incierto de las formas de lenguaje, de las experiencias, de lo previo. Y es incierto más todavía en medio de la situación de pandemia en la que nos desarrollamos. Para nadie ha sido fácil el proceso de educar en el mundo pandémico. Aprender las clases online, ser altamente creativos en las formas de comunicar los contenidos propuestos, invertir muchos recursos, tanto económicos, personales y anímicos, han constituido los espacios en donde lo pedagógico se ha ido desenvolviendo en medio de la emergencia sanitaria del COVID-I9. Pero, y es ahí donde volvemos con la idea que hemos ido proponiendo en estas primeras páginas, la entrega de contenidos pareciera no queda totalmente fecundada si no acompañamos esa entrega con una vida que es capaz de escuchar los relatos y la vida misma de los que se interrelacionan en el proceso de enseñanza-aprendizaje.

A través de la escucha, de la compasión, del cuidado y del mismo acompañamiento, como síntesis de las anteriores experiencias y de otras posibles, es que vamos propiciando que el acceso epistémico a los contenidos quede más fecundado. Enseñamos contenidos, pero también acompañamos esa enseñanza a través de una determinada forma de ser humanos. No acompañamos fuera de la vida, sino que es la misma vida el terreno que surge como herramienta y lugar fundamental del acompañamiento. Incluso podríamos proponer que desde ese vértice de lectura del mundo y posterior lectura de los contenidos, en cuanto entrecruce que vendría a propiciar al buen acompañamiento, se van desarrollando formas sugerentes de hospitalidad y cercanía humana. Recuperamos en esto lo propuesto por el filósofo español Josep María Esquirol quien nos dice: "hospedar y acoger significa dar refugio humano: no acogemos al otro en nuestras propiedades (casa y tierras), sino que lo acogemos en nuestra humanidad. La hospitalidad no nos hace perder raíces $\mathrm{y}$, sin embargo, nos hace más ligeros; nos aleja de las identidades estáticas y rígidas para actualizar el proceso de identificación" . Es interesante la figura del hospedaje y de la práctica de hospitalidad, en cuanto formas de acompañamiento, ya que parece que ellas surgen cuando la persona se encuentra en un estado de profunda

9 J.M. ESQUIROL, Uno mismo y los otros: de las experiencias existenciales a la interculturalidad. Herder: Barcelona, 20I7, p.37. 
crisis. Es en medio de la crisis donde la fragilidad de lo humano aparece como un elemento que, siendo propio, se va compartiendo con otros. Cuando la crisis humana, social, sanitaria e incluso pedagógica irrumpe en nuestra cotidianidad surge la vinculación de cuidado responsable con otros. Acompañar significa también estar con el que sufre la crisis, siendo capaz de escuchar su relato agónico y, desde allí, reconocer que en la propia vida la crisis también se da cita. De esta manera el acompañamiento viene a destrabar el egocentrismo al que, de alguna manera, todos tendemos. Acompañar es también dejarse acompañar por el acompañado, es aprender que el movimiento de compañía nunca es unidireccional, sino que en el encuentro las dos partes quedan transformadas en la formación y encuentro mutuo.

\subsection{El encuentro formado y transformado, clave en el proceso del acompañamiento}

Si anteriormente sosteníamos que la experiencia del acompañamiento tiene que ver con el encuentro mutuo realizado, vivido, celebrado, aprendido, un siguiente paso que hemos de propiciar es acentuar la dimensión de la formación en cuanto transformación, que, para nuestro caso, consideramos como una clave fundamental al momento de pensar el proceso del acompañamiento. Mantenemos, con ello, la clave de que al encontrarnos nos transformamos, y que dicha transformación supone el paso de condiciones egocentradas a una vinculación solidaria, hospitalaria y acompañante de los otros. En esta perspectiva, podemos rescatar, por ejemplo, la categorización que Paulo Freire propone al momento de hacer mención de lo que él denomina el profesor formador, el cual debe invitar a que sus estudiantes también sean formadores, es decir, el que ambos se puedan entrenar en la capacidad de dar sentido auténtico a lo que viven, accediendo primero al modo de vida con el que acceden a la comunidad educativa. Esto es llamado por Freire como "conocimiento relacional" ${ }^{\circ}$, el cual se comprende a partir de las siguientes intuiciones: "el conocimiento relacional, en el fondo interrelacional, "empapado" de intuiciones, adivinanzas, deseos, aspiraciones, dudas y miedos, al que no por eso le falta razón, no tiene la misma calidad que el conocimiento que se tiene del objeto aprehendido en su sustantividad por obra de la curiosidad epistemológica" "I. Lo indicado por Freire es sugerente en el sentido de que el comienzo de lo relacional no es el objeto estudiado, sino que son las vidas, la dimensión sutil del encuentro, los miedos y las dudas de los que acceden al vínculo pedagógico. Con esto, acompañamos

Io P. FREIRE, Pedagogía de los sueños posibles, p.63. II P. FREIRE, Pedagogía de los sueños posibles, p.63. 
no solo desde un determinado locus epistémico sino que acompañamos en el contacto vivencial. De hecho, ese es el comienzo de todo auténtico acompañamiento pedagógico.

Mismas intuiciones podemos encontrar en planteamientos como los del educador budista Daisaku Ikeda, es necesario reconocer cómo "nuestra vida cotidiana está colmada de oportunidades de aprendizaje, para nosotros mismos y para quienes nos rodean. Cada una de nuestras interacciones con los demás -diálogo, intercambio o participación-es un inapreciable estímulo para crear valor. Justamente porque aprendemos a partir del contacto con otras personas, el humanismo de un maestro es el factor clave de toda experiencia educacional" ${ }^{\prime 2}$. Con lo indicado por Ikeda, se vuelve a patentizar el sentido profundo del acompañamiento, en cuanto prácticas dialógicas, tendientes al intercambio y propiciadoras de la participación. Esto es interesante por lo siguiente: de alguna manera se deconstruye una visión del acompañamiento pedagógico meramente unidireccional. No es que el profesor y sólo el profesor acompañe pedagógicamente a sus estudiantes, sino que el acompañamiento surge a partir de una auténtica alianza política (comunitaria) entre los intervinientes del juego pedagógico. El mismo Ikeda expone dicha conciencia en los siguientes términos: "no soy el único en sostener que la vida de los alumnos no se transforma a fuerza de escuchar disertaciones, sino gracias al contacto estimulante con seres humanos. Por esta razón es tan importante el vínculo entre docentes y alumnos"ıs.

\subsection{La colaboración como espacio propicio del acompañamiento}

Por su parte, la pedagoga argentina Andrea Alliaud, para hablar del acompañamiento utiliza el término colaboración, e indica que esta categoría sumada a la de repetición e imaginación, constituyen los espacios a través de los cuales se forman los maestros que tienden a enseñar bien y a mejorar en su enseñanza. Hablando en particular de la colaboración (laborar con; nuevamente la lógica de la autotrascendencia, del ir-junto-con-otro), Alliaud indica que

"antes que la competencia, el trabajo colaborativo favorece la producción de una artesanía o un trabajo de buena calidad. La colaboración adquiere en esta propuesta un sentido particular, por cuanto favorece la productividad y la calidad de lo que se produce. Cuando desaparece la cooperación y predominan los solistas y la

I2 D. IKEDA, El nuevo humanismo. FCE: México, 2013, p.87.

I3 D. IKEDA, El nuevo humanismo, 88. 
competencia entre ellos, el trabajo se degrada" ${ }^{14}$.

Ahora bien, ¿qué significa que el acompañamiento o la colaboración puedan entenderse como dinámicas artesanales? Siento que es en esta pregunta y en su intento de respuesta donde surge una primera aproximación a pensar la teología como pedagogía del acompañamiento. En este tiempo de educación online, hemos tenido que mantener viva la creatividad como nota característica de nuestra acción docente.

Si antes la mecánica era una presencialidad aprendida, ahora y con la pandemia, los recursos, las formas, las dinámicas han debido reimaginarse y restructurarse. Eso es lo artesanal del quehacer pedagógico y teológico. Es la capacidad de salir de los límites que se presentan como normales (o más bien normalizados) y ser capaces de inaugurar una imaginación propositiva. Acompañar, pienso, es caminar de manera acorde a los tiempos. Es ser capaces de escuchar este tiempo, resignificarlo, dar mayor cabida a las emociones y a los sentimientos de quienes son nuestros primeros interlocutores, los estudiantes, y de aprender a trabajar con ellos. Al decir de Humberto Maturana, es generar el "danzar juntos en el conversar" "15 manteniendo siempre que la lógica del acompañamiento pasa por el establecimiento de una comunidad política que, sustentándose en el trabajo comunitario, es capaz de avanzar juntos en la consecución de un proyecto común. Esto, en palabras del mismo Maturana: "existimos como personas que viven en armonía psíquica y fisiológica, solo en un ámbito humano en el que se quiere convivir en la honestidad del respeto por sí mismas como el fundamento del bien-estar en el mutuo respeto" ${ }^{\prime \prime}$.

Pienso que, de alguna manera, el proceso de acompañamiento está en la lógica de lo sinodal como tarea de la Iglesia ${ }^{17}$. Si el sínodo es un camino junto a otros, dicho camino se vive mejor en la lógica del acompañamiento. La pedagogía debe aprender a caminar juntos

I4 A. ALLIAUD, Los artesanos de la enseñanza: acerca de la formación de maestros con oficio. Paidós: Argentina, 20I7), p.II4.

I5 H. MATURANA. Emociones y lenguaje en educación y política. Paidós: Santiago de Chile, 2020, p.I3.

I6 H. MATURANA. Emociones y lenguaje en educación y política, p.I4.

I7 La Comisión Teológica Internacional en el documento sobre la Sinodalidad en la Iglesia de 2018 , indicó que "la vida sinodal de la Iglesia se realiza gracias a una efectiva comunicación de fe, vida y compromiso misionero puesta en acción entre todos sus miembros. En ella se manifiesta la Communio sanctorum que vive de la oración, se alimenta de los Sacramentos, florece en el amor recíproco y hacia todos, crece en la participación de alegrías y pruebas de la Esposa de Cristo" (COMISION TEOLÓGICA INTERNACIONAL. La sinodalidad en la vida y en la misión de la Iglesia.

Ágape: Buenos Aires, 20I8, n.IIo (p.80). 
aún en medio de las situaciones de disenso. Es en este cruce creativo de experiencias en donde somos capaces de ubicar los trasfondos de una educación que sabe vivir los procesos personales y acompañarlos afectiva y efectivamente. La vida pedagógica y la teología deben estar preñadas de emocionalidad y de experiencias fuertes de encuentro.

\section{Resumen de los puntos anteriores y tránsito a pensar la teología como pedagogía del acompañamiento}

Los puntos que anteriormente hemos revisado, nos aportan algunos elementos comunes que, en este momento del artículo quisiera sintetizar. A partir de ellos, y hacia el final de este apartado, quisiéramos comenzar a pensar de qué modos la teología puede constituirse en una forma sugerente de lograr una pedagogía del acompañamiento.

En primer lugar, volver a la definición etimológica del acompañamiento, el cual se expresaba como la capacidad de estar con otros compartiendo el pan. Desde esta visión "nutricia", proponíamos que la misma nutrición no se quedaba solo en la capacidad de dar de comer alimentos físicos, sino que proponíamos una ampliación del mismo carácter nutritivo hacia las actitudes de la escucha, de la cercanía, de la solidaridad, de la compasión y de la misma pedagogía. A través de los procesos de enseñanza-aprendizaje vamos logrando un enriquecimiento vital que nos permite acceder a nuevas formas de comprensión de la realidad, de prácticas de transformación de la misma situación en la que nos encontramos y de promoción de todos aquellos con los que nos sentimos vinculados en medio de la trama de los acontecimientos.

A partir de ello, proponíamos que el comienzo de un sano acompañamiento pedagógico no era la comunicación de contenidos teóricos, sino que era la capacidad de poner la vida en común. Con Paulo Freire recuperábamos la importancia del movimiento de la lectura del mundo, elemento que antecedía - en sus palabras - a la lectura del texto académico. A través de la vida puesta en común, como primer momento delacompañamiento, vamos logrando una mejor interiorización de lo que son los participantes del proceso dialógico de lo formativo. A través de sus precomprensiones, de sus vidas, de sus formas de pensar, vivir y actuar, se va generando una mayor apropiación de los contenidos propuestos. Esto nos llevó, posteriormente, a adentrarnos en la importancia de generar la conciencia de cómo la formación (pedagógica) debe tener a la transformación humana. Esto, a su vez, tiende a la colaboración como forma sugerente del acompañamiento. 
Desde este marco pedagógico, procedamos a entrar en la segunda parte de este artículo, la cual estará destinada a pensar de qué modos la teología, como caso particular de lo pedagógico-formativo, puede constituir un modelo sugerente para pensar una pedagogía del acompañamiento. El modo de proceder en esta segunda parte será: en primer lugar, la recuperación de la experiencia pedagógica del autor de este artículo quien se desempaña como profesor de Teología en diferentes centros académicos chilenos y de qué ha significado hacer docencia teológica en medio de la pandemia del COVID-I9. A partir de este caso particular, se propiciará una reflexión tendiente a recuperar el carácter de compañía de toda teología. Como indicábamos en la primera parte del artículo, son las crisis humanas, sociales, sanitarias, culturales $\mathrm{y}$ eclesiales las que invitan a que los interactuantes en el proceso pedagógico asuman formas creativas por medio de las cuales puedan (y podamos) desarrollar de manera significativa nuestros procesos de acompañamiento y formación teórica. Por lo tanto, esta segunda parte propone un diálogo testimonial-analítico y, desde allí, proponer algunas claves para pensar nuestra labor teológica y pedagógica. Finalmente, se propondrá una categoría para pensar la teología como pedagogía del acompañamiento, a saber, la anástasisgogía, la cual hunde sus raíces en la experiencia de la Resurrección de Jesús y de cómo ella permite generar una forma sugerente de acercamiento, compañía y transformación para los interactuantes en la práctica pedagógica.

\section{La teología como pedagogía del acompañamiento: el caso particular de la experiencia pedagógica en el tiempo de pandemia}

\section{I El contexto en el cual se enmarca la reflexión: la docencia teológica en pandemia}

El día 03 de Marzo del 2020, la historia sanitaria reciente de Chile cambió totalmente. La pandemia del coronavirus representó y representa una forma de vida expuesta a la incertidumbre, al dolor y a la enfermedad con su clara posibilidad de muerte. La misma pedagogía y su quehacer, y en el contexto de la pandemia, se han visto transformadas en un grado no menor. La realización de clases online a través de diversas plataformas, la situación concreta de las familias de deber adoptar y adaptar sus espacios domésticos como verdaderas salas de clases, sumado al desgaste psico-emocional de los miembros de las comunidades educativas ${ }^{18}$, son

I8 En varios estudios se ha indicado que los profesores han presentado altos niveles de estrés y sobrecarga laboral durante la pandemia. Por ejemplo, en una noticia de Septiembre del 2020, se dieron a conocer los resultados de dos encuestas, realizada por la Fundación Chile y por la 
instancias en las cuales el modo de ejercer la pedagogía se ha visto fuertemente alteradas.

Actualmente realizo docencia en la Facultad de Teología de la Pontificia Universidad Católica de Chile y en el Centro Universitario Ignaciano de la Universidad Alberto Hurtado. En dichas unidades académicas, doy cursos en el área de la Formación General. Desde dicha áreas se ofrecen a todos los estudiantes de pregrado de la Universidad distintos cursos teológicos, de los cuales deben escoger e inscribir algunos por temas de créditos académicos. Esta inscripción y participación se realiza en alguno de los años de estudio de los estudiantes. Dichos cursos están conformados por estudiantes provenientes de distintas carreras, estudiantes que poseen distintas opciones de pensamiento, de vida y de creencia. Por lo tanto, dichos cursos son esencialmente heterogéneos. Pienso que este elemento es el que hace más llamativo el trabajo teológico, en cuanto la teología no solo dialoga con personas cuyas experiencias están en tu mismo registro teórico o creyente. Al proponer docencia teológica en estos espacios formativos, el docente va trabajando con miradas más críticas, con otras más desinteresadas, y con otras más abiertas a la fundamentación de sus opciones creyentes. Actualmente desarrollo cursos enmarcados en la Teología Fundamental, en la Antropología Teológica Fundamental y en la Cristología Fundamental. A través de ellas intento mostrar la importancia del diálogo, del ejercicio pedagógico de la pregunta-respuesta, de buscar cuáles son los intercisos culturales y sociales que permiten desarrollar una inteligencia de la fe, y de proponer una búsqueda adulta de la fe que vaya superando el mero porque es así o porque Dios lo dice.

\subsection{Los primeros desafíos y la conciencia de ofrecer compañía y elementos teóricos}

Desde aquí, vino el primer desafío. A comienzos de la segunda quincena de Marzo 2020 debía estar en mi ciudad y pensar cómo era el modo de la educación online. Habíamos aprendido de memoria el riguroso ritual de llegar a la sala, saludar, hacer la clase y finalizarla. Ahora, el espacio era otro, el rito, más o menos el mismo, pero el ánimo era nuevo. Esta es nuestra primera pandemia y, por ende, todos estamos todavía - aprendiendo a trabajarla. Nadie estaba - y pienso que todavía

instancia Educación 2020 (ambos organismos son chilenos). En los resultados de ve claramente que los profesores chilenos consideraban que el desgaste se presentó en niveles muy altos. C. SAID, "Profesores no dan más: el $57 \%$ dice que siente un desgaste extremo por la crisis", (2/09/2020) en https:/www.latercera.com/nacional/noticia/profesores-no-dan-mas-el-57-diceque-siente-un-desgaste-extremo-por-la-crisis/GWUMLXXHTZGGDLUHRHFM3TZ5DQ/ 
no lo estamos - preparados para el nuevo trabajo. De alguna manera las formas en las que llevábamos nuestra rutina, quedaron trastocadas fuertemente por la experiencia de la pandemia del COVID-I9. Ella nos recordó, nos recuerda y confío nos recordará el sentido más profundo de lo que es la vida humana: una realidad expuesta a lo incierto, al dolor, a la enfermedad, a la muerte pero también a la profunda esperanza, a lograr formas de creatividad y de repensar los proyectos que cada uno de nosotros hemos ido pensando a lo largo de este tiempo pandémico.

Con el desarrollo de la docencia teológica en pandemia, he ido aprendiendo una cuestión fundamental, a saber, que en este tiempo crítico, no por entregar más contenido teológico provocas más resonancia en personas que están o no conectadas con nuestra disciplina. Parafraseando a Fernando Berrios - que a su vez se inspira en Karl Rahner - hemos de trabajar en base a tres elementos: "el arraigo en la experiencia, la búsqueda del fundamento y la toma de conciencia sobre el contexto" ${ }^{\text {I. }}$. Estos elementos, a juicio de Berrios, constituyen "un triple desafío teológico y pedagógico" ${ }^{20}$. La educación teológica, creo, debería comenzar siempre desde esta experiencia, de este horadar la realidad social pero también personal, terreno en el cual buscamos el fundamento o el sentido de nuestras prácticas y de una toma de conciencia clara del contexto en el cual nos ubicamos y en el cual podemos hacer el discernimiento para la experiencia de Dios. Por ello el vínculo establecido entre teología y pedagogía es multidimensional. En palabras de Joaquín Silva: "la relación pedagogía-teología no se determina unidireccionalmente, sino que se resuelve por una relación que, siendo crítica, es de reciprocidad, de mutuo intercambio, de inclusión" ${ }^{21}$.

Por lo tanto, uno de los desafíos más relevantes que la teología y de la pedagogía en pandemia debe ser volver a trabajar directamente con las experiencias cotidianas de los interlocutores del proceso pedagógico. Como indicábamos al comienzo de nuestro artículo, es necesario volver al primer momento del acompañamiento, a saber, el ser capaces de poner en común las vidas de los intervinientes en el quehacer pedagógico. Es desde ellas y no fuera de ellas que el trabajo de conocimiento, interpretación, juicio y transformación de la realidad se va realizando de manera más fecunda. Por ello la importancia del horadar la realidad y,

I9 F. BERRIOS, "Pedagogía en teología: el aporte de Karl Rahner", en Veritas 26 (Marzo 20I2), pp. I87-I96, p. I9I.

20 F. BERRIOS, "Pedagogía en teología", p.I9I.

2I J. SILVA, "Pedagogía de la enseñanza teológica. La condescendencia de Dios como clave hermenéutica fundamental para la enseñanza teológica", en Teología y Vida VOL LI (2010), pp. 233-255, p. 234. 
en ella, de ser capaces de horadar nuestras propias vidas, reconociendo cómo ellas y en ellas se dan situaciones de crisis, de esperanzas, de proyectos, de vida y de muerte.

El teólogo argentino Nicolás Panotto posee intuiciones similares cuando piensa el desafío de construir, en su caso, una teología y una educación teológica que tenga a la niñez "como clave epistemológica"22. Panotto cree que la construcción de una teopética es clave al momento de recuperar otros saberes que permitan la reimaginación de una pedagogía teológica. Tres claves son reconocibles en su propuesta: en primer lugar, la necesidad de ahondar en los temas fundamentales de la teología, colocando especial énfasis en la forma de entender y hablar de Dios. Es el trabajo de la experiencia que poseemos en torno a lo sagrado y cómo las metáforas e imágenes en torno a dicha dimensión están presentes en la vida concreta de cada persona. En segundo lugar, la importancia de pensar nuevas mediaciones hermenéuticas, en las que lo lúdico y lo narrativo estén en la base de dicha mediación. Esto tiene que ver, con esta búsqueda del contexto y de sus posibles herramientas. En tercer lugar, pensar, dice Panotto, la niñez (yo añado la juventud por mi caso particular), como "epicentro del quehacer teológico, o sea, en las currículas, en las lecturas exegéticas, en la teología sistemática"23.

En el trabajo que personalmente he podido hacer, trato de generar espacios donde las preguntas - incluso más que las respuestas - sean las protagonistas del modo de acompañamiento. El acompañamiento, pienso, es una cuestión en donde las interrogantes son aquella condición de posibilidad de un buen estar-con. Y la mejor forma de captar esas preguntas es reconocer y escuchar mucho las interrogantes de los estudiantes. Berrios y Panotto comienzan desde la misma idea: el reconocimiento de las experiencias. Ese es el terreno de cultivo del acompañamiento. Por ejemplo, en la experiencia del acompañamiento espiritual, el acompañante no puedo obligar al acompañado a responder o actuar de una determinada manera. Se debe generar lo que en pedagogía se denomina espacio pedagógico protegido el cual define el medio o lugar donde ocurre la interacción pedagógica y que debe posee elementos intencionados que permitan que los participantes se sientan cómodos al momento de comenzar el descubrimiento de ellos mismos (acompañamiento espiritual) y el descubrimiento de un nuevo conocimiento (experiencia pedagógica).

22 N. PANOTTO. De juegos que hablan de Dios: hacia una teología desde la niñez latinoamericana. Sociedades Bíblicas Unidas: Quito, 2016, p.97.

23 N. PANOTTO. De juegos que hablan de Dios... p.98. 
Por lo tanto, ¿Qué elementos debería asumir, por tanto, la pedagogía teológica o la teología como pedagogía del acompañamiento? En primer lugar, la consideración de que Dios es el modelo del que acompaña, del que provoca, que dialoga y busca generar encuentro con los seres humanos. Si el acompañamiento no reconoce que el encuentro es un elemento clave al momento de entender el camino-junto-conotros, en ese momento el acompañamiento termina desintegrándose. No es un solipsismo, sino que es una alianza entre los acompañantes y los acompañados, entre los profesores y los estudiantes. Generamos alianzas y formamos un nosotros pedagógica y teológicamente situado. Esta es la lógica de la condescendencia divina, tal y como la reconoce Joaquín Silva quien la define como "clave hermenéutica fundamental para la enseñanza teológica"24.

En segundo lugar, la importancia del diálogo. El diálogo constituye un espacio pedagógico apropiado. A través de él confrontamos nuestras propias experiencias con aquellas situaciones que se nos comunican. Lo interesante de este proceso es que puede acontecer el llamado conflicto cognitivo, es decir, la situación en la cual una persona "verifica" que su conocimiento previo al nuevo aprendizaje entra en crisis debido al nuevo tema que se está conversando y aprendiendo. Por medio de dicho conflicto tenemos la posibilidad de ampliar nuestros modelos de pensamiento y nuestras pistas de actuación. De hecho, el acompañamiento invita a vivir una profunda humanización, proceso en el cual el diálogo adquiere un importante sentido.

José Asensio comenta: "lo constitutivo del diálogo es el saberse en una relación profundamente humana por cuanto tiene de reconocimiento del otro y de desafío a nuestras propias convicciones. Dialogar significa participar de una comunicación que, conviene decirlo, se produce entre personas y no entre ideas, y menos aún entre máquinas que no contestan" 25 . En la época de la pandemia, el diálogo que una teología que quiera entenderse como pedagogía del acompañamiento, debe transitar por aquellos elementos que dan sustento a la vivencia de la misma pandemia. ¿Qué significado tiene el dolor? ¿la muerte? ¿la ausencia y la distancia? ¿Dios mismo en la pandemia? ¿qué futuro? ¿lo cierto y lo incierto? ¿cómo has vivido la pandemia? ¿qué elementos has aprendido a rescatar? ¿qué es lo que más te ha costado de hacer, comprender o vivir en este tiempo? Son las preguntas las que nos adentran en la comprensión del mundo, del devenir humano y de la presencia de Dios

24 J. SILVA. "Pedagogía de la enseñanza teológica", p.233.

25 J. ASENSIO. Una educación para el diálogo. Paidós: España, 2004, p.I87. 
en nuestros entramados. Las preguntas representan una fuerza sugerente para ir repensando cómo realizar nuestras prácticas pedagógicas. Una pedagogía sin preguntas no es una auténtica tarea de transformación, ya que es desde las cuestiones más profundas desde donde vamos accediendo al sentido de lo que somos, de lo que hemos sido y de cómo imaginamos el querer ser.

Por ello, estos conceptos son los que, pienso, han dado sentido también a mi propia actividad pedagógica, en cuanto resignificación de las preguntas tradicionales pero puestas en perspectiva del mismo tiempo, de este entrecruce de pasado, presente y futuro, de un anhelo de posibilidades que, en medio de las situaciones de dolor y crisis de sentido, se pueden abrir como pequeñas lámparas que orientan y dan sentido al camino que se va emprendiendo.

En tercer lugar, la espiritualidad y lo místico. Para autores como Raimon Panikkar insiste en que la mística no es más que la apreciación y valoración de la vida . Si asumimos la definición panikkariana, la pedagogía y la teología deben ser disciplinas que apelen a lo biófilo más que a lo tanático, entendidos como dos modelos antropológicos que asumen ciertos modos de actuación. Una teología que quiera ser pedagogía del acompañamiento, debe asumir la vida como un elemento central de su propia constitución y estructuración. La espiritualidad para la vida y que alienta el surgimiento de la vida, es una vivencia de aquella fuerza de Dios que dio vida al primer Adán (Cf. Gn 2,7). La espiritualidad de la teología que acompaña es capaz de discernir dónde habita el Espíritu, sabiendo que Él en sí mismo camina por distintos recorridos (Cf. Jn 3,8). La espiritualidad y la mística de la pedagogía que acompaña debe ser una forma de vivencia de la fe con ojos abiertos, oídos despiertos, pies descalzos y manos extendidas; con una recuperación de elementos fundamentales para este tiempo y para todo tiempo.

\section{La anástasisgogía: provocación final con un concepto}

Quisiera finalmente proponer un concepto que, quizás, puede representar un espacio para repensar nuestra docencia teológica en este tiempo: la anástasisgogía. El concepto es la unión de la Anástasis (de la Resurrección de Jesús y del sentido del verbo griego $\alpha v \alpha \sigma \tau \alpha \sigma \iota \sigma$ ponerse de pie, acción de levantarse) y, por otra parte, la pedagogía como acción de conducir. Es un concepto que estoy ensayando en la perspectiva de mi trabajo doctoral que aborda la relación entre la Resurrección de Jesús y la antropología teológica fundamental. 
¿Qué puede significar este concepto? La Anástasisgogía supondría una pedagogía del aprender a colocarnos de pie, pero específicamente a dejar que otro nos ayude a levantarnos, a pasar de condiciones de inhumanidad y pecado a una vida auténtica, a vivir un proceso de acompañamiento. Por ejemplo, Jesús con Magdalena en Juan 20, practica una anástasisgogía, en cuanto la va conduciendo por medio de un método mayéutico (pregunta-respuesta) para el mayor conocimiento del amor. El teólogo francés Jean-Daniel Causse reconoce que el acontecimiento de la Pascua es el "nacimiento de una nueva subjetividad", de un origen nuevo en el corazón de la historia a partir del concepto de la resurrección. Ahora bien, la resurrección no acontece como situación abstracta, sino que posee una singular dimensión histórica y performativa. Dice Causse: "la muerte y resurrección de Jesús no tienen un valor de verdad más que convirtiéndose incesantemente en un acontecimiento que dé origen a un nuevo sujeto, del mismo modo que inauguran una nueva conciencia histórica” . La anástasisgogía vendría a dar origen a un nuevo sujeto. Causse habla de la emergencia de una nueva subjetividad gracias al contacto con el radicalmente Otro. La renovación que surge con la resurrección invita a recuperar el memorial subversivo del Cristo muerto y resucitado.

Patricio Alarcón, desde la pedagogía, propone el concepto de intimidades míticas como vinculación con el otro, reconociendo en él también un sentido espiritual. Dice Alarcón: "intimidad de goce cósmico o conexión de la individualidad con la trascendentalidad, de la parte con el todo, de lo personal con lo trascendental, intimidad de conexión con el silencio menor y mayor, que permite a partir de la toma de conciencia de existir de sí mismo de lo otro y de los otros, coalimentar el espíritu y el alma de los interactuantes". El acompañamiento es la vivencia de un goce estético, pedagógico, del eros, del alma, de la vida. Es una intimidad que merece respeto y acogida, silencio y palabra, reconocimiento y conocimiento.

Quizás, la Anástasisgogía es una pedagogía amante de la vida, una experiencia transformadora. La Anástasisgogía está en búsqueda de una renovación radical de la propia subjetividad. Por ello es antropológica, en cuanto la renovación acontece gracias al encuentro con el otro. La Anástasisgogía trabaja sobre la herida y la vulnerabilidad del ser humano debido a la vulnerabilidad del mismo Cristo crucificado. Sólo se comprende auténticamente la Resurrección cuando somos sensibles ante dicha fragilidad. Este es un concepto que aún debe trabajarse más y que, puede orientar futuras reflexiones. 


\section{Conclusiones en camino}

Al finalizar estas reflexiones, surgidas en medio de la crisis sanitaria del COVID-I9, crisis que no solo afecta a la salud personal, sino que acentúa desafíos profundos a lo social, a lo político, a lo cultural y, en nuestro caso, a lo pedagógico y a lo teológico, quisiera propone algunas conclusiones en camino. Los siguientes puntos no buscan ser consideraciones definitivas, sino que intentan ser una recapitulación y una apertura a futuras propuestas que puedan ayudar a pensar nuestras prácticas pedagógicas y teológicas.

I. En primer lugar, la consideración de una pedagogía que no solo encuentre en la transmisión de contenidos el corazón de su quehacer. En el tiempo de pandemia, en medio de la enseñanza remota, de las sesiones ZOOM con sus pantallas apagadas, con los micrófonos muchas veces en silencio, con la incertidumbre de saber si existen reales interlocutores detrás de los nombres, surge la importante consideración de asumir que las necesidades personales, la escucha, el estar, el repensar la presencia y la presencialidad, son elementos que dan sentido a lo que hemos propuesto como acompañamiento. Una pedagogía atenta al contexto, es aquella que es capaz de reconocer sus limitaciones y potencialidades. El contexto desafía nuestra creatividad y las formas de comunicar los contenidos que buscamos enseñar, pero también invita a que seamos capaces de poner nuestra vida a la escucha de otras vidas, vidas en las cuales, y desde una mirada de fe, reconocemos al que es el autor de toda existencia.

2. En segundo lugar, la importancia de hacer partícipes a los estudiantes y demás interlocutores de nuestras propuestas pedagógico-teológicas. Si el acompañamiento es ser capaces de partir-compartir el pan con otros (desde su etimología), dicha acción supone la presencia de una comunidad en la que se va viviendo la dinámica del estar-con. En el acompañamiento, además, vamos haciendo responsables a otros de sus propios procesos. De esta manera, esta mirada pedagógica supone un evitar una suerte de paternalismo o de conductismo que impide el propio proceso. Si con los autores que hemos revisado en esta propuesta reflexiva hemos accedido a la consideración de que es en medio de la comunidad, y de un modo particular, de una comunidad de vulnerables donde mejor hacemos experiencia del acompañamiento, es importante que la teología como forma particular de la pedagogía del acompañamiento, sea capaz de acentuar el carácter de reconocimiento de esa comunidad y de proponer, por ejemplo, el sentido teológico de 
pensar y vivir con sentido la vulnerabilidad. La pandemia, situación crítica de nuestro tiempo, nos ha recordado a todos el sentido más real de la vulnerabilidad corporal, espiritual, pedagógica, social y política. En ella hacemos experiencia del encuentro entre vulnerables y es ahí donde, pienso, las distintas formas de acompañamiento tienen un sentido mayor, sentido tendiente a compartir con otros nuestros relatos vitales, de proyecto y de espiritualidad.

3. Finalmente, la importancia de mantener la profunda creatividad al momento de vincular interdisciplinarmente nuestras distintas áreas de conocimiento. En esta propuesta pedagógica hemos pretendido realizar un diálogo entre pedagogía y teología a partir del enfoque del acompañamiento. Pienso que nuestro tiempo pandémico, de crisis de instituciones, de búsquedas de nuevas formas de comprender la realidad en la que estamos inmersos, exige de parte de nuestros constructos epistémicos la audacia de ser capaces de aportar distintas herramientas de interpretación y explicitación de la realidad. Esto también puede ser una forma sugerente de acompañamiento, en el cual las distintas disciplinas científicas aportar sus preguntas e intentos de respuestas a los problemas detectados. Esto, pienso, surge como un desafío posterior a las intuiciones que hemos pretendido aportar en este artículo. 
